\title{
Aprendizaje de estrategias para aplicación de ciencia en la educación médica
}

Juan M. Muñoz Cano, Jeannette Ramírez Mendoza, Karina E. Hernández Abreu, y Juan A. Córdova Hernández División Académica de Ciencias de la Salud, Universidad Juárez Autónoma de Tabasco. Avenida Méndez 2838-A, Tamulté, C.P.86150. México. (correo-e: juan.munoz@ujat.mx, ceoodrajanne@hotmail.com, drakarinaabreu@gmail.com juan.cordova@ujat.mx

Recibido Dic. 24, 2019; Aceptado Feb. 21, 2020; Versión final Abr.1, 2020, Publicado Ago. 2020

\begin{abstract}
Resumen
El objetivo de este artículo es describir prácticas que se dirigen a que los estudiantes aprendan a acceder, usar y elaborar información. Este es un estudio analítico de las actividades realizadas en clases de licenciatura en medicina. La evaluación de los cursos fue de tipo formativa, dirigida a la identificación, evaluación y aplicación de datos para resolver preguntas clínicamente estructuradas y situaciones problema. Los estudiantes podían identificar y revisar el contenido conceptual en revisiones sistemáticas, estudios clínicos controlados y estudios de cohortes en casa y resolver las situaciones problemáticas en clase que se revisaban constantemente como evaluación formativa. Con estas técnicas, los estudiantes fueron capaces de desarrollar estrategias para aplicar la ciencia en forma de evidencia para resolver problemas de salud. Estas experiencias son minoritarias en el currículo por lo que se requiere desarrollarse en más materias para capacitar a médicos capaces de aprender de forma permanente y autónoma.
\end{abstract}

Palabras clave: educación médica; educación científica; innovación educativa; aula invertida

\section{Learning strategies for science application in medical education}

\begin{abstract}
The purpose of this research is to describe practices designed to help students learn how to access, use, and develop information. This is an analytical study of the activities performed in medical undergraduate classes. The evaluation of the courses was of formative type, aimed at the identification, evaluation and application of data to solve clinically structured questions and problem situations. Students identified and reviewed conceptual content in systematic reviews, controlled clinical studies, and cohort studies. They also solved problem scenarios in class that were constantly reviewed as formative evaluation. The results show that students were able to develop strategies to apply science in the form of evidence to solve health problems. These experiences are a minor part of the curriculum. Therefore, it is concluded that more subjects incorporating the practices designed in this study are required to train doctors to make them capable of learning permanently and autonomously.
\end{abstract}

Keywords: medical education; scientific education; educational innovation; flipped classroom 


\section{INTRODUCCIÓN}

El curriculum tradicional de la educación médica, no sólo en México sino en las instituciones donde se estructura en bloques, con asignaturas canónicas, presenta serios obstáculos para convertir la medicina en disciplina científica. Éstos se estructuran en cuatro aspectos. 1) El problema de la transposición didáctica de los datos científicos que se encuentran en artículos primarios en bibliotecas virtuales hacia los textos tradicionales de enseñanza en las aulas (Chevalard, 1998). 2) La persistencia de métodos y técnicas de enseñanza que no tienen sustento científico. 3) La naturaleza de la ciencia que la configura como una construcción social. 4) La ciencia requiere de un contexto de aplicación, por lo tanto, es necesario el aprendizaje de estrategias de localización y selección de datos para la transferencia del conocimiento médico científico a la práctica profesional de los futuros médicos.

Con el propósito de superar esos obstáculos los autores han elaborado una estrategia para la gestión del conocimiento a partir de la revisión de fuentes de información primaria, guías clínicas, revisiones sistemáticas y normas oficiales mexicanas. Pero la gestión de los recursos requiere de la identificación de las situaciones en las cuales se necesita su aplicación. El diagnóstico contextual tanto de las comunidades como de los casos individuales se basa en la lectura de tablas epidemiológicas, problemas de salud pública y de tendencias en los patrones de consumo y sus consecuencias. En la última parte se describen resultados de las prácticas escolares en las cuales se introducen innovaciones.

\section{La educación médica en el curriculum tradicional}

La mayor parte de las instituciones de educación médica del mundo se encuentran conformadas en dos bloques emanados de las observaciones del informe Flexner (Irbi et al., 2010). El básico en el cual se enseñan conceptos que se espera que los estudiantes memoricen para comprender el siguiente. El clínico en el cual se espera que los estudiantes apliquen lo que memorizaron años antes. Esta disposición que estructura el curriculum no sólo de medicina sino de la mayor parte de las profesiones parte de la idea ingenua de que si se hace memorizar conceptos de la ciencia (en forma de definiciones, esquemas, y otros) antes que los contenidos conceptuales y procedimentales de la profesión. Se piensa que los estudiantes los aplicarán una vez que se encuentren, ya en el tercer año de su trayectoria escolar, en las cuestiones que, en el bloque clínico deberán memorizar a su vez (en forma de definiciones, listado de signos y síntomas, entre otros). Esto no sucede de esa manera idealizada. Las quejas de los docentes de los cursos superiores, acerca de la velocidad del olvido de los contenidos de los primeros cursos, es habitual (Rodríguez, 2014; Torres et al, 2011), aunque no suficiente para modificar la estructura organizacional que en algunas universidades se basa en estructuras independientes de la educación médica en forma de departamentos, sean de morfología, bioquímica o fisiología, que tienen sus propios objetivos.

Por el contrario, los datos científicos muestran que las actividades de memorización acrítica no producen aprendizaje. Mientras que las prácticas tradicionales parten de la idea de que el conocimiento se puede transmitir por el docente y adquirir por el alumno, los hallazgos de las neurociencias muestran la complejidad de las estructuras que se movilizan para el aprendizaje pero que requieren de un hacer para conformar el aprendizaje duradero (Academia Nacional de Ciencias de Estados Unidos [ANCEU], 2000: capítulo 1). La manera tradicional de tratar de hacer científica la educación médica, con cursos de ciencia y no mediante el aprendizaje de la aplicación de la ciencia en la clínica fracasa en sus intenciones e incluso esos conceptos, memorizados de manera enciclopédica, no adquieren sentido (Granados, 2009) y se describen como intrascendentes para la práctica (Watmough et al, 2010).

Estas percepciones, ideas, preconceptos con los cuales se justifica el modo tradicional de sistema escolar, obstaculizan que se aprenda a problematizar y, peor, que se aprenda a producir conocimiento acumulable con base en datos de estudios que se apliquen a la experiencia cotidiana. Por ejemplo, la práctica de una medicina sustentada en la tradición hace hace persistir la Reacción de Widal (Jaramillo et al., 2012) para el diagnóstico de la fiebre tifoidea a pesar de que ya no se le considera prueba diagnóstica. Otro ejemplo es el uso de la biometría hemática para evaluar el desarrollo de infecciones, cuando deben usarse la procalcitonina o la proteína $C$ reactiva, lo cual es perceptible si se analiza la fisiología de los leucocitos y su capacidad para migrar a través de las paredes vasculares. De aquí es claro que las escuelas de educación superior, no sólo las de medicina, no tienen como objetivo la que debe ser su principal función: enseñar a los estudiantes a aprender para el logro de la capacidad del aprendizaje permanente, autónomo y autorregulado, como posición ética (AMM, 2020)

\section{Obstáculos del curriculum tradicional al aprendizaje de la ciencia}

La primera dificultad para transitar este camino es la creencia que ser médico se circunscribe a elaborar recetas, prescribir medicamentos de la manera en cómo lo realizan los que ya están en la práctica y que no 
son necesarias capacidades para lectura y escritura de manera compleja, autónoma. Esto es consecuencia, en parte, de que la escuela no es capaz de proporcionar significatividad a los cursos, sean de ciencias básicas o de clínicas con lo que de manera paradójica se fomenta el desprecio hacia las ciencias como objeto de estudio (Duit, 2006) de manera particular y a los cursos escolares de manera general pues es frecuente escuchar que los mismos docentes afirman: "se aprende medicina en el hospital".

El resultado es que se piensa que los pacientes sólo son caras difusas que acuden por una prescripción y cuyas características individuales y contextuales no son relevantes para proporcionar recetas ya que sólo estarán ante el médico si al caso cinco minutos. Por lo contrario, ser médico requiere, y debería ser explícito en todas las escuelas de medicina, de la capacidad de elaborar diagnósticos, cuya falla fue la tercera causa de muerte en Estados Unidos en 2013 (Makary y Daniel, 2016). Además, es necesaria la disposición para consolar y confortar, sin que una capacidad exima de la otra.

La capacidad para elaborar los diagnósticos es crítica ya que "hay pocas posibilidades de prescribir los remedios apropiados si uno se equivoca de diagnóstico" (Godet, 1995) por lo que se convierte en una importante causa de demandas en Estados Unidos (Medscape, 2015). Esto es lo que conforma la variabilidad clínica ya que mientras en algunos centros la resolución es alta en otros es muy pobre porque no en todas las unidades clínicas hay preocupación del uso de datos de literatura científica disponible, a pesar de la existencia de metaanálisis, estudios clínicos controlados, estudios de cohortes, que sustentan las guías de la práctica clínica. Esta deficiencia ha de afrontarse en las escuelas de medicina mediante el aprendizaje en el contexto de la práctica de una Medicina con Base en Datos (Evidence-Based Management) (Kepes et al., 2016) así como la Educación Médica Basada en Datos (Best Evidence Medical Education) (Sánchez, 2012). Pero dada la complejidad de las bases de datos esto puede caer en otra pose más, como los resultados de innovaciones curriculares como la educación con base en competencias.

Para disminuir el riesgo el profesor debe convertirse en el experto en la gestión del conocimiento. Esto porque de su experticia depende el que los estudiantes aprendan un proceso de búsqueda cada vez más complejo donde se guía a los estudiantes hacia la autonomía. También es importante analizar el contexto de los estudios primarios, pues de ello depende un mayor nivel de calidad de la información. De esa manera, al elevar los estándares de la información, es posible gestionar aplicar y difundir conocimientos altamente relevantes (Kepes et al., 2016).

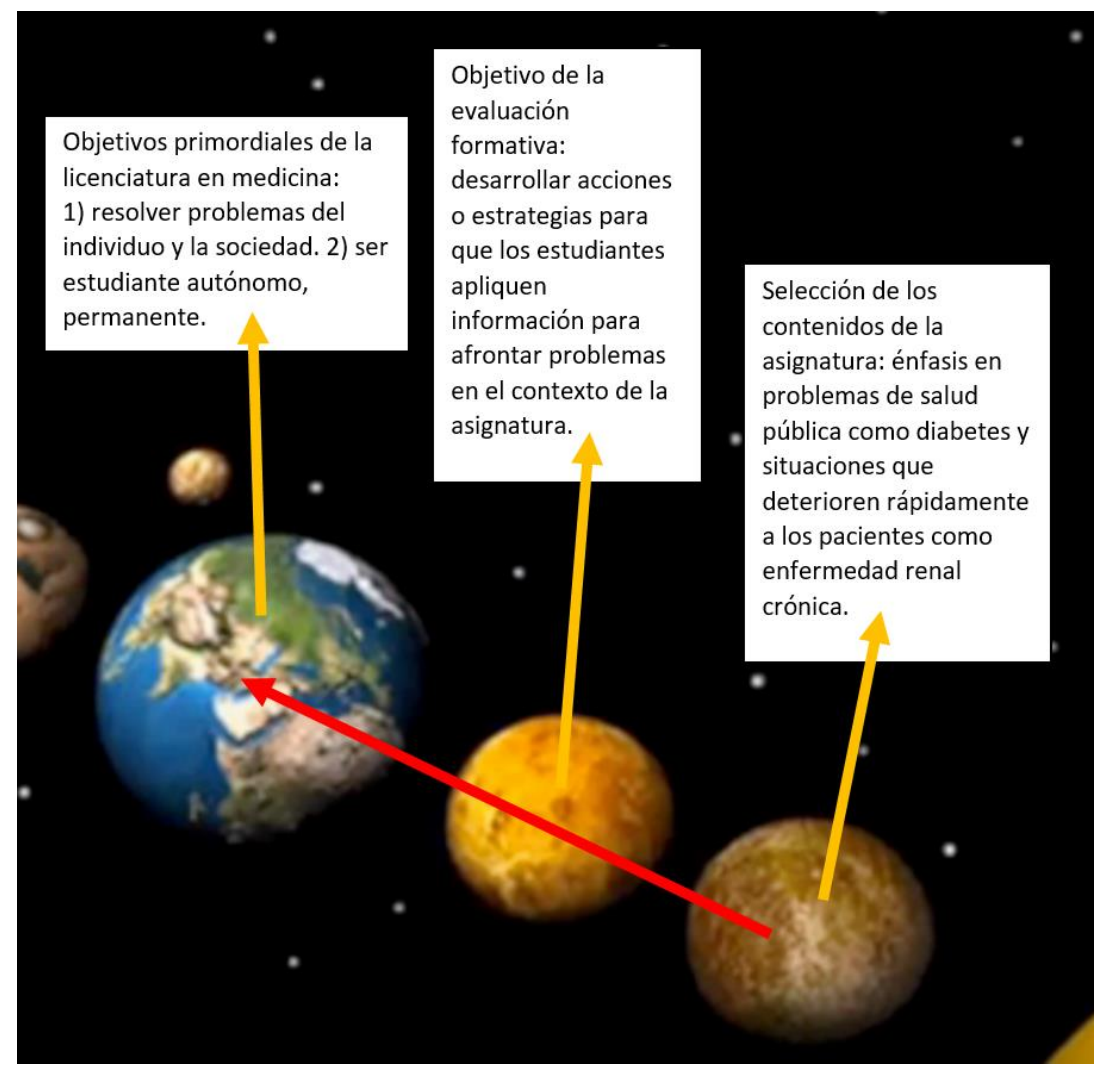

Fig. 1. Alineación de contenidos y evaluación con los objetivos de la licenciatura. Los contenidos se seleccionan de manera que se dirijan al logro del objetivo de la licenciatura. 
Es relevante formar en la escuela las capacidades necesarias para la identificación de los perfiles epidemiológicos de la región geográfica en que se desempeñará. Para ello debe aplicar competencias de lectura y escritura básicas del estudiante que ingresa a la universidad (Pérez, 2008). Es necesario construir tanto la capacidad de problematizar como la de gestionar los recursos conceptuales para responder a los problemas identificados: 1) ¿Cómo es la realidad inmediata (como enfermedades o factores de riesgo) sobre la que se quiere intervenir? 2) ¿Qué factores contextuales la condicionan? 3) ¿Cuál es el juicio o evaluación que se hace de la situación problema (que no necesariamente es sencilla o unívoca)? 4) ¿Cuáles son los recursos y medios operacionales (información) que se disponen para actuar en función de la resolución de los problemas y/o la satisfacción de las necesidades o carencias detectadas (de los pacientes)? 5) ¿Cuáles son los factores más relevantes que probablemente han de actuar de manera positiva, negativa o neutra, con relación a los objetivos o finalidades propuestas dentro del plan de manejo a realizar (con los pacientes)?

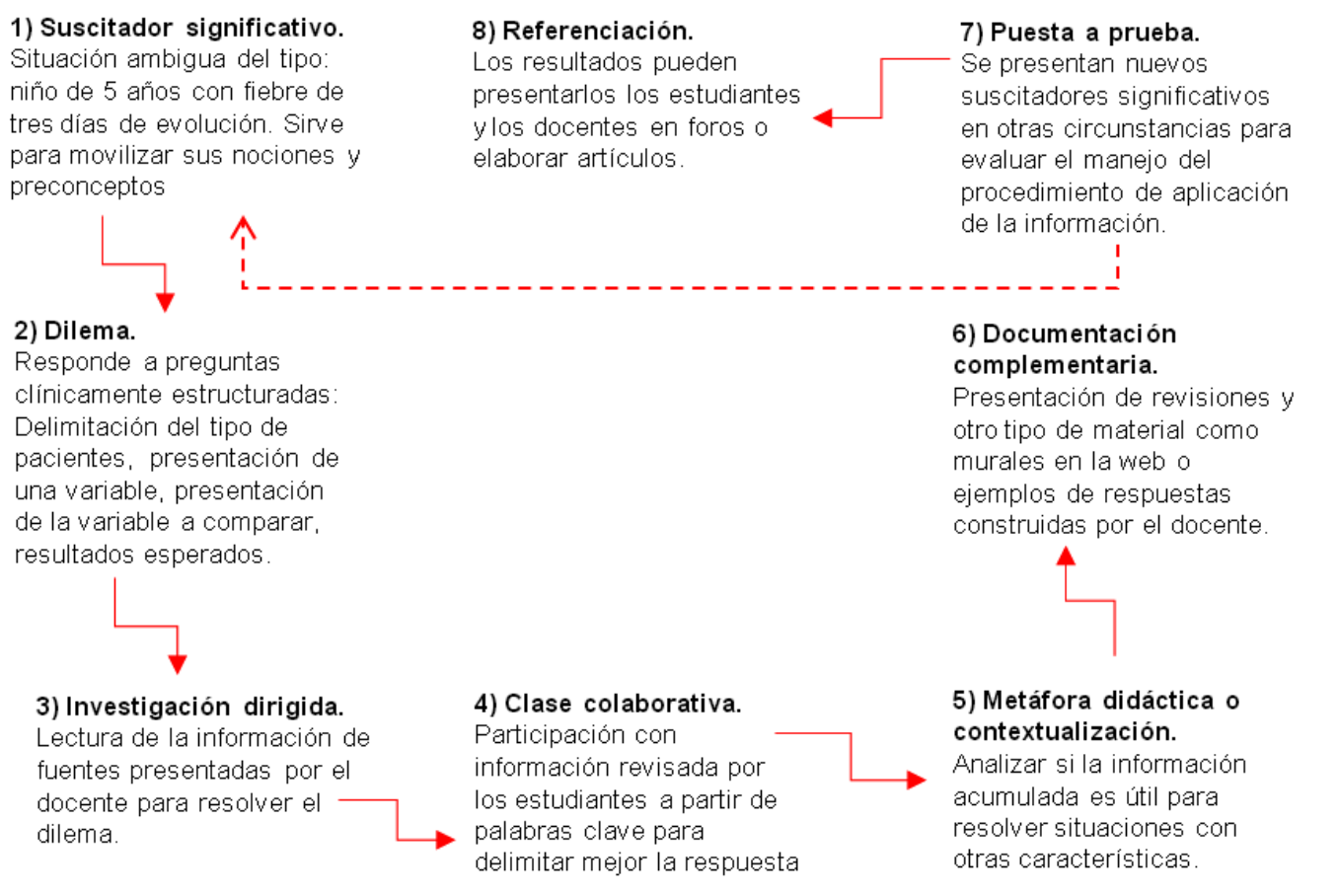

Fig. 2. Secuencia didáctica empleada. Nótese que en los puntos 5 y 7 de la secuencia se puede trabajar conceptualmente a mayor profundidad.

\section{METODOLOGÍA}

Es un estudio analítico del trabajo escolar de los semestres de 2015 a 2018 y de 2019 en su primer semestre. Las asignaturas fueron "Fisiopatología", "Endocrinología, metabolismo y nutrición" y "Proyecto de investigación" cuyo diseño se elaboró con acuerdo a una clase alineada (Figura 1). También se alineó a la Misión explícita de esta Universidad: resolver problemas de salud y desarrollar capacidades de aprendizaje permanente y autónomo.

La estrategia para las actividades escolares se centró en revisar conceptos en casa y elaborar las tareas en el aula en forma de clases invertidas (Espinosa et al., 2018; Moya y Williams, 2016) con apoyo de una plataforma en línea. En las clases invertidas "el papel del profesor en la sala es el de auxiliar a los estudiantes, y no el de transmitir información" (Bergmann y Sams, 2012). Se desarrolló en una secuencia didáctica con los siguientes elementos: 0) Ubicación curricular, 1) Suscitador significativo, 2) Dilema, 3) Investigación dirigida, 4) Clase colaborativa, 5) Metáfora didáctica o contextualización, 6) documentación complementaria, 7) Puesta a prueba, 8) Referenciación (Figura 2).

Para integrar los contenidos de una asignatura con conceptos de otras, se les presentaron a los estudiantes situaciones problema, por ejemplo, en forma de preguntas clínicamente estructuradas, donde se deben identificar contenidos desde el nivel de salud pública hasta el molecular necesarios para su resolución (Figura 3). A diferencia, una situación problema o suscitador significativo es ambigua por lo que se deben movilizar más contenidos de un problema específico. Los contenidos conceptuales se seleccionaron con 
acuerdo a los que los estudiantes tenían que saber, en este caso investigar, para solucionar la situación problema específica a las unidades de aprendizaje. Los contenidos procedimentales son los que los estudiantes deberían saber para identificar, jerarquizar y analizar la información. Los contenidos valorales se dirigieron a acatar diversas normas como la Ley General de Salud, su reglamento, la Norma relativa al expediente clínico, el llamado "Juramento de Hipócrates", en realidad de la Asociación Médica Mundial, en su versión de 2017, ya que el médico debe ser consciente que la medicina se desarrolla en ambientes de meta-cultura.

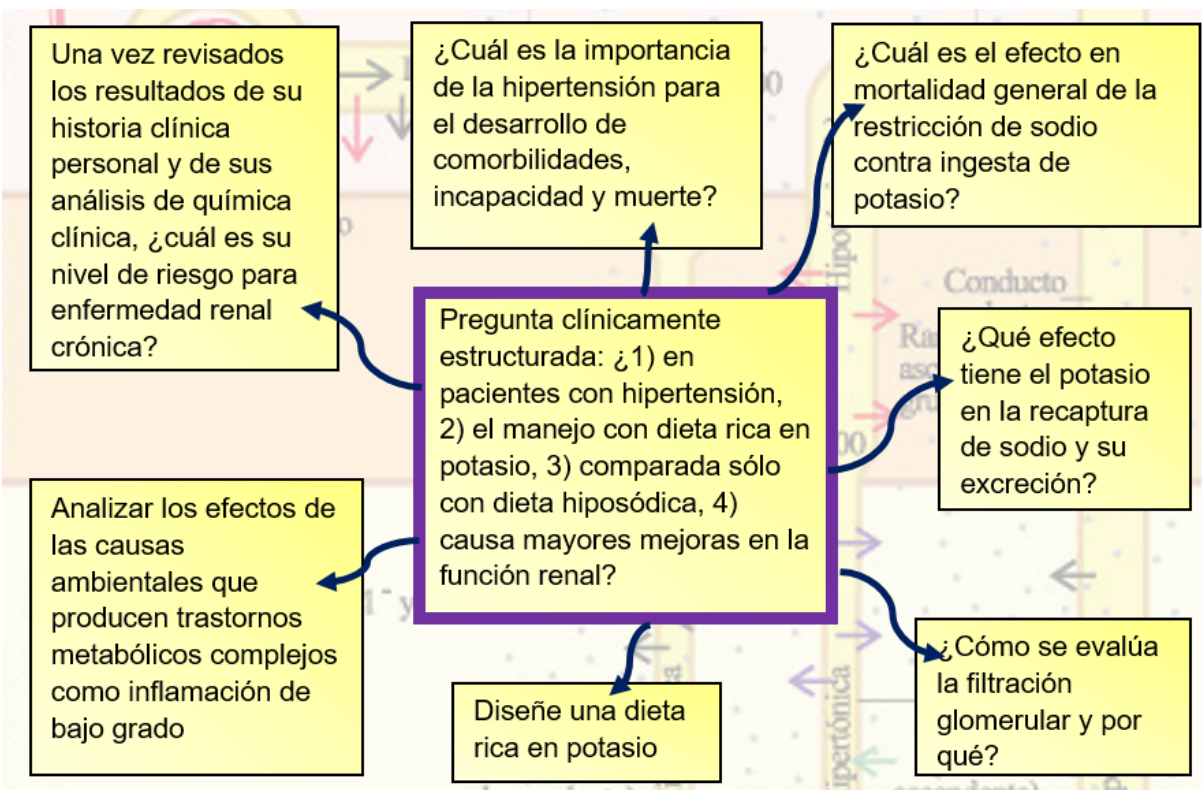

Fig. 3: El modelo de trabajo permite el análisis en niveles de mayor profundidad, desde salud pública, fisiología, fisiopatología, hasta nivel molecular.

La evaluación se sustentó en el paradigma "la evaluación formativa en la enseñanza diferenciada" (Rueda, 2018, p. 170). Para ello se elaboraron rúbricas para guiar a los estudiantes en la elaboración de las respuestas a los ejercicios planteados en el aula con acuerdo a la a evaluación formativa y como requisitos formales para la acreditación (Barriga, 2009, pp. 150-151). La evaluación formativa se llevó a cabo en la revisión de cada sub-meta de las actividades consideradas como objetivos del proyecto consistente en: 1) Resolución de dilemas y toma de decisiones clínicas, 2) Elaboración de conclusiones acerca de un producto o intervención, 3) Elaboración de cuadros comparativos, 4) Elaboración de hipótesis de diagnóstico (Figura 4).

\section{Ruta}

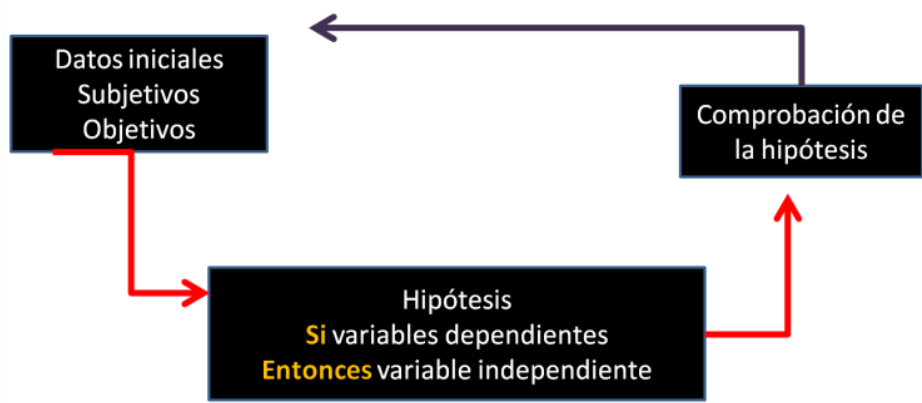

Fig. 4: Construcción de hipótesis de diagnóstico. Nótese que la comprobación de la hipótesis se hace mediante datos, sean clínicos, laboratorio o imagen.

Otros instrumentos de evaluación fueron el diario del estudiante y el portafolio de evidencias. Para el diario se les solicitó que escribieran las preguntas que surgieron en la clase durante la realización de las tareas para resolver mediante la búsqueda de información, sea en las lecciones o en nueva literatura. Para el portafolio de evidencias se revisaron las aportaciones de los estudiantes en el aula virtual del curso. De esta manera se hizo una práctica escolar reflexiva, dirigida a resolver las situaciones problema mediante la búsqueda de artículos científicos. Esta información de evaluó de manera crítica y se sustituyó por otra de 
más calidad en su caso (Melchor, 2011), con acuerdo a las rúbricas. Así, la evaluación continua se centró en construir las competencias para formar estudiantes autónomos y permanentes.

Como evaluación sumativa se elaboró un cuestionario objetivo estructurado que integra propedéutica, fisiopatología, terapéutica, así como las ramas principales de la medicina, pediatría, ginecología y obstetricia, medicina interna. La referenciación se realizó sistemáticamente mediante la presentación en foros y la publicación de artículos científicos.

\section{RESULTADOS}

Al analizar el cuaderno del estudiante se trabajó tanto en base a las anotaciones que deberían investigar ya que se trataba de conceptos que no conocían, como de otras búsquedas. Se encontró que muchos de los estudiantes se limitaron a escribir lo que se anotaba en el pizarrón para ilustrar algún aspecto de las discusiones y a escribir las preguntas guía que surgieron durante las actividades presenciales. Hubo al principio falta de búsqueda de datos para sustentar las clases con base en literatura científica, pero se logró que cada vez mayor número de estudiantes tuvieran acceso a datos de calidad.

Al revisar los diarios de los estudiantes se encontró que tuvieron dificultades para estructurar actividades postclase de calidad (Tabla 1). Se encontraron transcripciones de los artículos que se les recomendaron e incluso varios presentaron información extra. Al inicio la mayor parte de los estudiantes sólo transcriben conceptos e ideas principales, de manera habitual. Por ello no se mostraba como un trabajo de integración, análisis de información contradictoria, o aplicación para situaciones específicas. En general $20 \%$ de los estudiantes lograron aplicar información a sus recomendaciones para los pacientes mencionados en los problemas (Tabla 2).

Tabla 1. Análisis de las anotaciones en el diario de los estudiantes

\begin{tabular}{|c|c|c|}
\hline Temática & Anotaciones & Observaciones \\
\hline \multirow[t]{6}{*}{$\begin{array}{l}\text { Tratamiento no } \\
\text { farmacológico } \\
\text { de las } \\
\text { enfermedades } \\
\text { no transmisibles }\end{array}$} & $\begin{array}{l}\text { El tratamiento en el estilo } \\
\text { de vida tiene un impacto } \\
\text { limitado en la obesidad }\end{array}$ & $\begin{array}{l}\text { Incorrecto. La evidencia } \\
\text { muestra que con medidas } \\
\text { como beber solo agua y hacer } \\
\text { un desayuno grande sin } \\
\text { modificar las calorías modifica } \\
\text { el perfil metabólico }\end{array}$ \\
\hline & $\begin{array}{l}\text { Beber leche con bajo } \\
\text { contenido de grasas y } \\
\text { agua en vez de gaseosas } \\
\text { o bebidas azucaradas }\end{array}$ & $\begin{array}{l}\text { La recomendación de leche } \\
\text { se hace por el aporte de } \\
\text { calcio. Para México debe } \\
\text { insistirse en las tortillas ya que } \\
\text { se hacen a base de maíz } \\
\text { integral nixtamalizado }\end{array}$ \\
\hline & $\begin{array}{l}\text { Ratones a los que se les } \\
\text { implanta flora bacteriana }\end{array}$ & $\begin{array}{l}\text { Las bacterias no son parte del } \\
\text { reino flora. Son microbiota }\end{array}$ \\
\hline & $\begin{array}{l}\text { Se recomienda la triada de } \\
\text { lácteos, cereales y fruta } \\
\text { que se complementa con } \\
\text { alimentos de origen animal }\end{array}$ & $\begin{array}{l}\text { La evidencia muestra que se } \\
\text { debe disminuir el consumo de } \\
\text { alimentos de origen animal, } \\
\text { incluso lácteos }\end{array}$ \\
\hline & $\begin{array}{l}\text { Hay relación entre la } \\
\text { microbiota con menor } \\
\text { probabilidad de obesidad, } \\
\text { síndrome metabólico y } \\
\text { diabetes }\end{array}$ & $\begin{array}{l}\text { Información de calidad. } \\
\text { Artículo primario }\end{array}$ \\
\hline & $\begin{array}{l}\text { El consumo de granos } \\
\text { enteros disminuye } \\
\text { significativamente } \\
\text { colesterol, glucemia y } \\
\text { mejora la señalización para } \\
\text { apetito y saciedad }\end{array}$ & $\begin{array}{l}\text { Información de calidad. } \\
\text { Artículo primario }\end{array}$ \\
\hline
\end{tabular}

Aunque tenían escrito en sus cuadernos resúmenes, fragmentos e ideas centrales de artículos científicos, no usaban esa información para sustentar un punto de vista. Los estudiantes se mostraban dispuestos a 
buscar en la red, pero sólo de manera plana, quizás con la idea de que tienen acerca de la lección escrita para la exposición con diapositivas.

Para la elaboración de las tareas en el aula, en un caso se partió de palabras clave para la búsqueda de información que sustentara porqué comer durante el día y no la noche. Una de las palabras clave fue "circadiano". Algunos estudiantes identificaron a una hormona como significativa para dicha recomendación. A partir de ese dato se les colocó información en un muro en Padlet suficiente para que pudieran establecer con mayor precisión y evidencia sus prescripciones a pacientes para un estilo de vida saludable.

Tabla 2. Reportes de los estudiantes para la recomendación "No saltarse el desayuno, debe ser grande".

\begin{tabular}{lll}
\hline & Sustento de la recomendación & \multicolumn{1}{c}{ Observaciones } \\
\hline Estudiante A & $\begin{array}{l}\text { Debe ser completa y equilibrada } \\
\text { (más abundante con relación a la }\end{array}$ & $\begin{array}{l}\text { Usa palabras vacías como } \\
\text { comida y la cena) }\end{array}$ \\
& un uso preciso. Faltan sus \\
& fuentes de información. No \\
& sustenta la evidencia que \\
& permite hacer \\
& recomendaciones en \\
& ocasiones contrarias a los \\
& paradigmas del personal de \\
& nutrición.
\end{tabular}

\begin{tabular}{|c|c|}
\hline $\begin{array}{l}\text { Estudiante B } \\
\text { (fragmento) }\end{array}$ & $\begin{array}{l}\text { En quienes no desayunan } \\
\text { aumenta la leptina pero se } \\
\text { produce resistencia en los } \\
\text { receptores centrales por lo cual } \\
\text { deja de tener función como señal } \\
\text { de saciedad [6]. En niños que } \\
\text { desayunan el desarrollo escolar } \\
\text { es mejor y tienen menos factores } \\
\text { de riesgo cardiovascular y de } \\
\text { síndrome metabólico } \\
\text { independientemente de la calidad } \\
\text { de la dieta [7], esto sucede } \\
\text { también en mujeres que no } \\
\text { desayunan en las cuales es más } \\
\text { probable la hipertensión arterial } \\
\text { [8]. }\end{array}$ \\
\hline
\end{tabular}

Se emplean las referencias insertadas en el muro, además otras de las páginas en la red de Univadis y Medscape, entre otras. Para identificar las fuentes primarias de estas páginas se usaron palabras clave. síndrome metabólico de la dieta [7], esto sucede desayunan en las cuales es más [8].

A partir de preguntas clínicamente estructuradas los estudiantes aprendieron a elaborar premisas con base en información científica conclusiones acerca de cuestiones relevantes para la práctica médica, tanto referentes al uso de medicamentos y procedimientos validados como de la oferta de tratamientos con base en magnetoterapia o células madre.

\section{DISCUSIÓN}

En los procesos de educación en los esquemas tradicionales se pueden identificar al menos tres problemas generales que obstaculizan que la evidencia científica se utilice como conocimiento para la solución de problemas de la vida real: 1) La dificultad para que los conceptos científicos puedan ser aprendidos de la mejor manera posible. 2) Los procesos educativos deben estar sustentados en el conocimiento científico de cómo se aprende, 3) Revalorar tanto la diversidad cultural como la ciencia como construcciones humanas.

Para que los conceptos científicos puedan ser aprendidos de la mejor manera posible se presenta la necesidad de que el profesor debe tener experticia para la identificación de los sitios para colectar información científica. De otra manera no puede guiar a sus estudiantes (Figura 1, Tabla 1). La segunda es que la interpretación de los estudiantes debe ser lo más parecida a la que los científicos confieren a sus datos, lo que se denomina el conocimiento sabio (Chevallard, 1998, p. 12). Esto, que también se conoce como saber erudito es lo que se encuentra en las revistas de investigación y en las Guías clínicas (Alumno B, Tabla 2).

Por lo contrario, en el modo tradicional de enseñar ciencia se espera que, al proporcionar un contexto de aplicación, los estudiantes aprendan más los conceptos (Norman, 2009). Este modelo de trabajo escolar muestra desconexión entre lo que se enseña, contenidos de un programa de ciencia, y el contexto. Por ello 
se debe analizar primero la situación problema para seleccionar los conceptos de ciencias que se requieren con el propósito de resolverla (Figura 4). La puesta en marcha de este tipo de proceso educativo demuestra que se construyen las bases de las competencias transversales: 1) resolver problemas clínicos, 2) aprender cómo adquirir conocimiento, 3) aprender a cuidar a los pacientes, 4) trabajo en equipo, 5) manejo de tecnología, 6) investigación clínica (Kebede y Pronovost, 2015; Pershing y Fuchs, 2013).

Los cursos tradicionales, por lo contrario, se crearon en la lógica de que, "para aprender cabalmente la nutrición" es necesario aprender primero bioquímica, biología molecular y fisiología (Vega-Franco e Iñárritu, 2001). Peor, en el modo tradicional, centrado en el libro de texto y en la información suficiente para hacer una diapositiva, el acto de explicar ese conocimiento erudito a los estudiantes es fallido porque los docentes no tienen contacto con el conocimiento como tal sino a interpretaciones de los autores de los libros. Lo que se explica, repite y memoriza en las clases no necesariamente son los nuevos hallazgos. Con el argumento de que hacer un libro dificulta que sus contenidos se mantengan al día persisten ideas generales que deben ser superadas. El fenómeno denominado transposición explica por qué en libros de texto, libros auxiliares y en materiales didácticos diversos, los conceptos tienen más afinidad por las teorías socialmente construidas las cuales reciben un importante apoyo por los medios que con las teorías científicas (Alumno A, Tabla 2).

De esta manera persisten en las clases de las asignaturas clínicas errores conceptuales como que el dolor posterior al inicio de una sesión de ejercicio es debido al ácido láctico, que una función importante del colon es reabsorber agua, que al sentirse somnolientos a media mañana porque "se les baja la glucemia" es necesario beber una soda que está endulzada con jarabe de alta fructosa. La realidad es que el dolor es a causa del estrés oxidativo por lo que más ejercicio genera mayor cantidad de antioxidantes, que el agua se reabsorbe en el duodeno y la fructosa no eleva la glucemia ya que se metaboliza a ácidos grasos en el hígado.

Los procesos educativos deben estar sustentados en el conocimiento científico de cómo se aprende (ANCEU, 2000). Estos cursos se basan en neurociencia. 1) El enfoque de las actividades fue interdisciplinar. De esta manera es más probable que haya estudiantes que se motiven a realizar la tarea (Guillén et al, 2015). 2) Se trató de activar funciones mentales superiores mediante el aprendizaje de una estrategia para responder a dilemas: si se recomienda o no una subrutina de exploración clínica, una prueba de diagnóstico, un fármaco, unas recomendaciones para educación de los pacientes (Figura 1). 3) Al desarrollar experiencias diferentes a las de la mayoría de las asignaturas, se promovió la "inscripción de la experiencia". Esto porque no se trató de reproducir un texto o información sino de promover el pensamiento crítico, la imaginación y creatividad (nuevas búsquedas) de los estudiantes posteriormente a mostrarles el procedimiento en forma de investigación dirigida con lo que pudieron activar el sistema de neuronas en espejo.

Es necesario que los estudiantes se formen en la gestión del conocimiento y no en la memorización acrítica como base de la formación de las competencias transversales (Palés y Nolla, 2016). Revalorar tanto la diversidad cultural como la ciencia como construcciones humanas requiere de modificaciones en los procesos educativos. La reflexión acerca de las actividades docentes han de considerar lo que se llama la naturaleza de la ciencia. Esto no se circunscribe a los contenidos conceptuales ni se dirige hacia el estudio de los diferentes métodos o abordajes de los objetos de estudio. El conocimiento científico se construye mediante avances y retrocesos, sobre todo, de la labor constante que lo mejora. Los problemas que se plantean los científicos, las preguntas a las que responden, no se resuelven súbitamente. Por ello es importante que los estudiantes acepten que están en un proceso formativo, que no lo saben todo o al menos las respuestas a las preguntas del examen (Muñoz y Maldonado, 2013). En este sentido se responsabilizarán de formar las capacidades para ser estudiantes permanentes, autónomos, uno de los objetivos explícitos en la Misión de la escuela de medicina, necesario para el cumplimiento del otro: resolver problemas de salud del individuo y la sociedad.

La limitante ha sido evidente ya que para el caso de identificación de enfermedades y la elaboración de esquemas de tratamiento con apego a la evidencia científica se creó la Biblioteca Cochrane. Este es un sistema de revisiones sistemáticas de los reportes de investigación publicados acerca de problemas específicos y las prácticas sociales en que se aplican. Desde su creación se ha promovido precisamente como mecanismo de transponer el conocimiento erudito en documentos accesibles a los médicos, así como a los profesores y estudiantes de medicina. En las clases habitualmente se prefieren los libros que son reediciones de publicaciones del siglo XX. Esta es una tradición promovida por la industria de los libros escolares pues de trabajarse en el aula con base en la resolución de problemas y no en la explicación de temas en todo caso sólo se necesitaría un libro por docente y no un libro por alumno (Perrenaud, 2006, p. 79). 
A pesar de que constituyen información confiable, y que sus bases de datos se encuentran disponibles para México en la Biblioteca Virtual de Salud en la práctica educativa son poco promovidas como parte de las herramientas necesarias para la gestión del conocimiento. Como en los procesos que se mostraron en este trabajo, es importante el consultar resultados de estudios clínicos controlados, de cohortes, estudios de casos (Tabla 2). Por último, a pesar de que los estudiantes no se encuentran a gusto con el desarrollo de los programas educativos tradicionales (Muñoz y Córdova, 2017), hay una elevada tasa de abandono a las clases que se describen en este trabajo.

\section{CONCLUSIONES}

Los estudiantes desarrollan ideas de mayor complejidad a partir de conceptos que se encuentran en más de una fuente o texto, esto es base para aplicar la información científica a problemas de salud concretos. Estas experiencias son minoritarias en el plan de estudios de la licenciatura de medicina pues en la mayoría de los cursos se aprende mediante la exposición en clases, por lo que se requiere se desarrollen en más asignaturas para formar médicos capaces de aprender de manera permanente y autónoma. Por ello son necesarias para reconfigurar la estructura curricular para cerrar la brecha entre los planes y programas de instituciones de educación médica en modelo tradicional, y la información que se encuentra disponible y accesible en bases de datos y portales especializados.

\section{REFERENCIAS}

Asociación Médica Mundial (AMM)., Declaración de la AMM con normas para el mejoramiento continuo de la calidad de la atención médica. https://www.wma.net/es/policies-post/declaracion-de-la-amm-con-normas-para-el-mejoramientocontinuo-de-la-calidad-de-la-atencion-medica/ (2020).

Barriga-Díaz, A., El docente y los programas escolares. Lo institucional y lo didáctico. IISUE-UNAM, Ciudad de México. (2009).

Bergmann, J., y Sams, A., Flip your classroom: Reach every student in every class every day. Washington: International Society for Technology in Education. (2012).

Bransford, J., Brown, A., y otro autor, Cómo aprende la gente: cerebro, mente, experiencia, y escuela. National Academy Press. Washington DC Extracto Capítulo 1. Revista del Instituto de Matemática y Física, 44-64. (2000).

Chevallard, Y., La transposición didáctica. Del saber sabio al saber enseñado. Buenos Aires: Aique. (1998).

Chevallard, Y., Readjusting didactics to a changing epistemology, DOI: 10.2304/eerj.2007.6.2.131. European Educ. Res. J, 2(6): 131-134. (2007).

Duit, R., La investigación sobre enseñanza de las ciencias. Un requisito imprescindible para mejorar la práctica educativa. Rev. Mex. Invest. Educ. 30(1): 741-770 (2006).

Espinosa, T., Araujo, I. S., y otro autor. Aula invertida (flipped classroom): innovando las clases de física. Revista de enseñanza de la Física, 30(2), 59-73. (2018).

General Medical Council. (GMC), Tomorrow's Doctors. http://www.gmcuk.org/static/documents/content/TomorrowsDoctors_2009.pdf (2009).

Godet, M., Prospectiva: ¿por qué?, ¿cómo? Siete ideas claves. Traducido por Javier Medina V. Cuadernos de Administración, 14(21):156-164. (1995).

Granados J., La enseñanza de las ciencias básicas en medicina desde la perspectiva de la justificación epistemológica del currículo, DOI: 10.15517/revedu.v33i2.504. Educación; 33: 51-60 (2009).

Gregory, A., y Armstrong, R., Flaws in the fabric? DOI: 10.5694/mja12.c1001Med. J. Aust., 7(197): 361 (2012).

Guillen, J.C., Pardo, F., y otros tres autores, Principis neurodidàctics per a l'aprenentatge. Temps d'Educació, 49, 49-67 (2015).

Hernández, C., Aguilera, M.G., y otro autor. Enfermedades gastrointestinales, situación en México. Enf. Inf. Microbiol, 31(4):137-151 (2011).

Irby, D.M., Cooke, M., y otro autor, Calls for reform of medical education by the Carnegie Foundation for the Advancement of Teaching: 1910 and 2010, DOI: 10.1097/ACM.0b013e3181c88449. Acad Medicine; 85: 220-227 (2010)

Jaramillo, H.J., De la Peña J.A., y otros dos autores. Reacción de Widal en población sana de Mexicali. Sal. Pub. Mexico, 54(2):106-107 (2012).

Kalman, J., Discusiones conceptuales en el campo de la cultura escrita, DOI: https://doi.org/10.35362/rie460719. Rev. Iberoamericana Educación, 46: 107-134 (2008).

Kebede, S., Pronovost, P., It is time to reinvent the wheels of medical training, DOI: $10.1097 / \mathrm{ACM} .0000000000000600$. Academic Medicine, 90(2), 126 (2015). 
Kepes, S., Andrew, A., y otros dos autores. Evidence-based management and the trustworthiness of our cumulative scientific knowledge: implications for teaching, research, and practice, DOI: 10.5465/amle.2013.0193. Academy of Management Learning \& Education, 13(3): 446-466 (2016).

Maturana R., H. y Varela, G.F., El árbol del conocimiento. Buenos Aires: Lumen (2003).

Makary, M. A., y Daniel, M., Medical error-the third leading cause of death in the US. British Medical Journal, DOI: 10.1136/bmj.i2139 3;353:i2139 (2016).

Medsacape. Medscape Malpractice Report 2015: Why most doctors get sued. http://www.medscape.com/features/slideshow/public/malpractice-report-2015 (2015).

Moya, P., y Williams, C., Efecto del Aula Volteada en el rendimiento académico: Estudio comparativo basado en el resultado del rendimiento académico con metodología Aula Volteada y Clase Tradicional para la asignatura de Salud Pública. R.E.C.S., 13(1), 15-20 (2016).

Muñoz Cano, J.M., y Córdova Hernández, J.A., Percepción de pasantes de medicina en servicio social sobre su plan de estudios, https://doi.org/10.19136/pd.a28n63.2632. Perspectivas Docentes, 28(63), (2017).

Muñoz Cano, J.M., Maldonado Salazar, T.N.J. Dificultades para la construcción de la literacidad en la educación médica. Rev. Actual. Investig. Educ, 13(2), 1-18 (2013).

Norman, G., Teaching basic science to optimize transfer, DOI: 10.1080/01421590903049814. Medical Teacher, 31(9), 807-811. (2009).

Palés-Argullós, J., y Nolla-Domenjó, M., Competencias transversales, un tema pendiente en las facultades de medicina. F.E.M., 19(5), 227-228 (2016).

Pérez, G. Las producciones textuales de los estudiantes en el contexto universitario. Memoralia, 5(3):109-122 (2008).

Perrenaud, P., Diez nuevas competencias para enseñar. Querétaro, México, (2004).

Perrenaud, P., Construir competencias desde la escuela. Santiago de Chile: J. C. Sáenz. (2006).

Pershing, S., Fuchs, V.R., Restructuring medical education to meet current and future health care needs, DOI: 10.1097/ACM.0000000000000020. Academic Medicine, 88(12), 1798- 1801 (2013).

Rodríguez, R., Retos de las ciencias básicas en la educación médica. Gac. Med. Mex,150 (Supl 3): 358-360. (2014).

Rueda, M., La evaluación educativa: límites y desafíos. En: La educación y los retos de 2018. UNAM, Ciudad de México. (2018).

Sánchez-Mendiola, M., Educación médica basada en evidencias: ¿Ser o no ser? Investigación en Educación Médica, 1(2), 82-89. (2012).

Torres E., Osuna C. y otro autor, Reprobación en las carreras del área de ciencias de la salud de la Universidad Autónoma de Baja California, México. Educ Humanismo 13: 34-50 (2011)

Vega-Franco L., Iñárritu, M.C., La enseñanza de la nutrición en la carrera de medicina, Rev Fac Med UNAM, 44(5):224229 (2001).

Watmough S.D., O'Sullivan H., y otro autor, Graduates from a reformed undergraduate medical curriculum based on Tomorrow's Doctors evaluate the effectiveness of their curriculum six years after graduation through interviews. BMC Med. Educ. 10: 65 (2010). 